\title{
The Use of Platelet-Rich Plasma for Conservative Treatment of Partial Ulnar Collateral Ligament Tears in Overhead Athletes: A Critically Appraised Topic
}

\author{
Bradley J. Conant, Nicole A. German, and Shannon L. David
}

\begin{abstract}
Clinical Scenario: Rates of ulnar collateral ligament (UCL) injuries continue to rise in overhead athletes of all ages. Surgical interventions require minimally 6 months and up to 2 years of rehabilitation. Younger athletes and those with partial tears have seen positive results with conservative treatment approaches. Platelet-rich plasma (PRP) continues to be studied with various orthopedic injuries, and its use has the potential to improve return-to-sport rates and reduce recovery time. Focused Clinical Question: Do PRP injections improve conservative treatment outcomes in overhead athletes with partial tears of the UCL compared with conservative treatment alone regarding return to participation? Summary of Search, Best Evidence Appraised, and Key Findings: A literature search was performed to locate all studies investigating outcomes when PRP is included in a conservative treatment program for overhead athletes with partial UCL tears. Three case series qualified and were reviewed. Clinical Bottom Line: Current evidence suggests that including PRP in a conservative treatment program can improve outcomes in overhead athletes with partial UCL tears. Athletes whose treatment included PRP show higher return-to-competition rates and shorter recovery times compared with athletes who used rehabilitation alone. Athletes with grade- 1 and proximal-based grade- 2 injuries returned to competition at rates comparable with athletes undergoing surgical intervention. For optimal conservative management outcomes, PRP injections should be recommended for treatment of partial UCL tears. Strength of Recommendation: The studies qualifying for inclusion are level 4 evidence based on the 2011 Oxford Centre for Evidence-Based Medicine levels of evidence. The studies are well designed and show consistent results, but higher level studies need to demonstrate similar results to improve the body of evidence. The strength of recommendation is $\mathrm{C}$.
\end{abstract}

Keywords: injury management, sports medicine, rehabilitation, return to play, elbow

\section{Clinical Scenario}

The anterior bundle of the ulnar collateral ligament (UCL) is the primary stabilizer against valgus forces within the functional range of $30^{\circ}$ to $120^{\circ}$ of elbow flexion..$^{1-3}$ During the throwing motion, substantial valgus loads placed on the elbow during the late cocking and early acceleration phases often exceed what may be required to damage the ligament. ${ }^{1-3}$ Baseball pitchers are most often affected by UCL tears, with injury rates continuing to rise. ${ }^{1,4}$ Reconstructive surgery is the most common treatment approach for UCL tears, but increasing evidence shows that surgical repair and conservative treatment are viable options for certain athletes. A surgical repair of the ligament will require approximately 6 months of rehabilitation before returning to competition. ${ }^{5}$ If reconstructive surgery is necessary, rehabilitation will last at least 12 to 18 months..$^{1,4,6-8}$ Select populations have seen positive results, including shorter return-toplay times, with conservative treatment approaches consisting of relative rest and various modalities for pain control followed by structured rehabilitation including range of motion and progressive upper-extremity strengthening exercises before beginning an interval throwing program. ${ }^{3,4,7,9}$ More specifically, younger athletes presenting with lower grade, proximal-based, primary injuries have shown good outcomes with conservative treatment. 4,6,7,9 For most of these athletes, and athletes at all levels, the recovery time

The authors are with Health, Nutrition, and Exercise Sciences, North Dakota State University, Fargo, ND. German (nicole.german@ndsu.edu) and David (shannon. david@ndsu.edu) are corresponding authors. associated with surgical intervention is not feasible based on longterm goals or competition timelines. Improving outcomes for these athletes relies on reducing recovery time and increasing the likelihood of returning to competition.

Platelet-rich plasma (PRP) contains a variety of growth factors with laboratory research showing evidence of an accelerated healing process and improved tissue healing. PRP has been studied clinically in treatment of various orthopedic conditions as well., $, 6,7,10$ For athletes identified as good candidates for conservative treatment, including PRP in the treatment plan may improve healing of the UCL, improve return-to-competition rates, and minimize the time necessary to return compared with rehabilitation alone.

\section{Focused Clinical Question}

Do PRP injections improve conservative treatment outcomes in overhead athletes with partial tears of the UCL compared with conservative treatment alone regarding return to participation?

\section{Summary of Search, "Best Evidence" Appraised, and Key Findings}

- A literature search was performed to locate all studies investigating outcomes when PRP is included in a conservative treatment program for overhead athletes with partial UCL tears. For the purposes of the literature search, conservative treatment was operationally defined as any treatment plan that did not include surgical intervention. Typical conservative 
approaches included, but were not limited to, rest, pain control, pharmacologic intervention, rehabilitative exercise, and throwing programs.

- Three studies that met inclusion and exclusion criteria were located and included in the critically appraised topic. ${ }^{6,8,10}$ All 3 of the studies were case series.

- All 3 studies found reduced recovery times and improved return-to-sport rates with the use of PRP compared with rehabilitation alone. The studies also found promising results with nonprofessional athletes, the population most likely to attempt conservative treatment. No adverse effects were reported in any study. Patient selection and injury severity play a crucial role in treatment outcomes. ${ }^{6,8,10}$

\section{Clinical Bottom Line}

Current evidence suggests that the addition of PRP to a conservative treatment program may improve outcomes in overhead athletes with partial UCL tears. ${ }^{6,8,10}$ Athletes whose treatment included PRP show higher return-to-competition rates and shorter recovery times before returning to competition compared with athletes who performed rehabilitation only. ${ }^{3,4,6-10}$ Additionally, athletes with grade- 1 and proximal-based grade- 2 injuries return to competition at rates comparable with athletes undergoing surgical intervention. ${ }^{1,6,8,10}$ No side effects have been reported when including PRP in treatment, and its use does not preclude surgical reconstruction if conservative treatment fails. ${ }^{6,8,10}$ The decision to use a conservative treatment approach must be made individually, and clinicians should consider many athlete- and injury-specific factors. ${ }^{1}$ For optimal conservative management outcomes, PRP injections should be recommended for treatment of partial UCL tears.

\section{Strength of Recommendation}

The studies qualifying for inclusion in this critically appraised topic are low-quality evidence. Each study is level 4 evidence based on the 2011 Oxford Centre for Evidence-Based Medicine levels of evidence. The studies are well-designed case series and show consistent results, but a need remains for higher level studies including case-control and randomized controlled trials to demonstrate similar results to improve the body of evidence. The strength of recommendation is $\mathrm{C}$.

\section{Search Strategy}

\section{Terms Used to Guide Search Strategy}

- Patient/Client group: overhead athletes with partial tears of the UCL

- Intervention: PRP injections and conservative treatment

- Comparison: conservative treatment without PRP

- Outcomes: ability to return to sport and to previous level of competition

- Time: length of time before returning to participation

\section{Sources of Evidence Searched}

- PubMed

- MEDLINE (Clarivate Analytics)

- MEDLINE (EBSCO)

- SPORTDiscus
- Cochrane Library

- Reference Lists

\section{Inclusion and Exclusion Criteria}

\section{Inclusion Criteria}

- Overhead athletes

- Partial tear of the UCL

- Conservative treatment with PRP injections

- Original research articles

- English language

\section{Exclusion Criteria}

- Additional treatment not found in a "traditional" conservative treatment plan

- Surgical repair or reconstruction of the UCL

\section{Results of Search}

The literature search revealed 156 unique articles. Of these, 3 qualified for inclusion in this critically appraised topic. All 3 qualified studies were case series and represented level 4 evidence based on the 2011 Oxford Centre for Evidence-Based Medicine levels of evidence. ${ }^{6,8,10}$ The search process is shown in Figure 1 and the search results are summarized in Table 1.

\section{Best Evidence}

The studies summarized in Table 2 represent the best evidence available based on the inclusion of PRP in a conservative treatment program for overhead athletes with partial tears of the UCL. All 3 articles were determined to be level 4 evidence based on the 2011 Oxford Centre for Evidence-Based Medicine levels of evidence.

\section{Implications for Practice, Education, and Future Research}

As the number of young athletes experiencing damage to the UCL and subsequent UCL repair or reconstruction continues to rise, efforts to develop effective conservative treatment protocols should increase. ${ }^{1}$ The impact of surgical recovery times relative to most athletes' career length cannot be overstated, with repair requiring approximately 6 months of recovery and reconstruction typically requiring 12 to 18 months. ${ }^{1,4-8}$ Although complete tears of the UCL almost universally require surgical repair or reconstruction, there is a growing body of evidence suggesting that nonsurgical approaches can successfully treat partial tears in some patients. ${ }^{6,8,10}$ This critically appraised topic sought to determine if traditional conservative treatment approaches could be improved with the addition of PRP.

Current research investigating the effects of PRP on UCL tears shows positive outcomes related to return to competition and length of time missed. These studies report $73 \%$ to $96 \%$ of athletes returned to competition with an average of 11 to 12 weeks of time missed. ${ }^{6,8,10}$ These findings are notable increases from previously reported outcomes ranging from $42 \%$ to $84 \%$ of athletes returning to competition and a minimum average recovery time of 


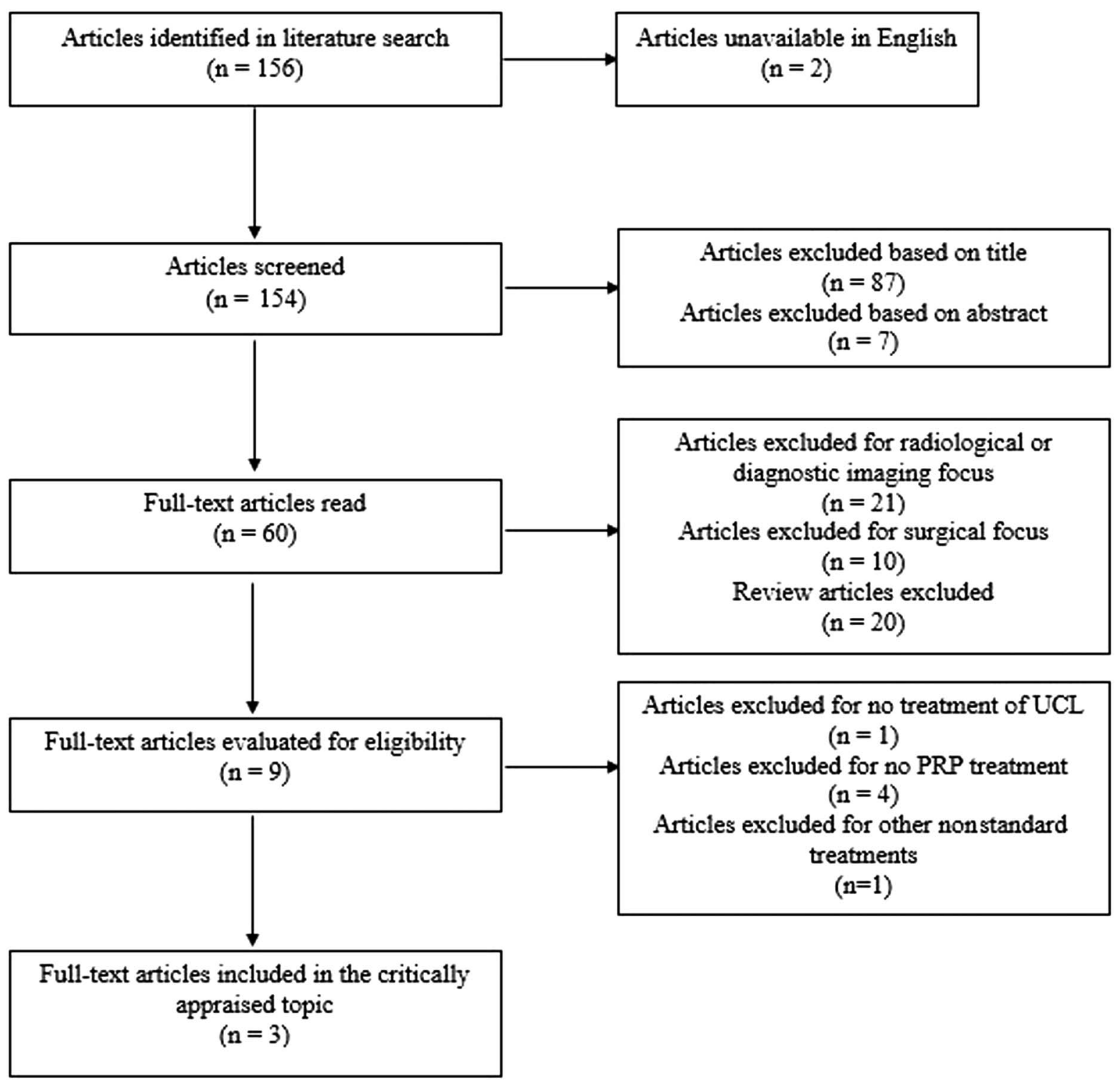

Figure 1 - Search strategy. PRP indicates platelet-rich plasma; UCL, ulnar collateral ligament.

Table 1 Summary of Study Designs of Articles Reviewed

\begin{tabular}{|c|c|c|c|}
\hline Level of evidence & Study design & Number reviewed & References \\
\hline 4 & Case series & 3 & Deal et al, ${ }^{10}$ Dines et al, ${ }^{6}$ and Podesta et $\mathrm{al}^{8}$ \\
\hline
\end{tabular}

24.5 weeks. $^{3,4,7,10}$ Importantly, the studies also demonstrated that inclusion of PRP treatments in a conservative treatment plan does not preclude surgical reconstruction for those athletes who are unable to return to competition. ${ }^{8,10}$

Practicing clinicians must consider several factors when considering treatment plans for an athlete based on these results. Appropriate patient selection is crucial to the success of conservative treatment, and clinicians must educate each patient to give a realistic perception of the likelihood that treatment with PRP will be successful. Patients demonstrating diffuse damage or a lower grade partial tear of the UCL on magnetic resonance imaging are more likely to respond to conservative treatment than those with a higher grade partial tear or complete tear. ${ }^{4,6,7,9}$ Similarly, better results are seen in patients with proximal-based tears compared with those with distally based tears.,6,7,9 Patients who have previously undergone surgical reconstruction or repair should not be expected to respond favorably to conservative treatment. ${ }^{10}$

The patients in the reviewed studies are mostly high school and college athletes, which are 2 populations for whom avoiding surgical intervention may be most important. ${ }^{6,8,10}$ Clinicians should be mindful of the timelines and career goals of these athletes when weighing options and should be aware that positive results have been demonstrated in these groups. The results of these studies are most applicable to baseball athletes, with the vast majority of athletes included in both the PRP groups $(91 \%)$ and rehabilitation only groups (94\%) being baseball players. ${ }^{6,8,10}$ These 
Table 2 Characteristics of Included Studies

\begin{tabular}{llll}
\hline & Deal et al ${ }^{\mathbf{1 0}}$ & Dines et al & \\
\hline Study design & Case series & Case series & Podesta et al ${ }^{\mathbf{8}}$ \\
Participants & Consecutive series of 25 patients & Forty-four baseball players diag- & Thirty-four healthy overhead athletes with a \\
& with grade-2 medial UCL injuries & nosed with UCL insufficiency by & symptomatic grade-1 or grade-2 partial \\
& with positive moving valgus & physical examination and con- & UCL lesion diagnosed by physical exam \\
& stress test and/or milking & firmed by MRI who had failed a & and confirmed by MRI. All patients had \\
& maneuver and MR arthrogram, & conservative treatment program & failed at least 2 mo of nonoperative treat- \\
& desire to return to sport, ability to & of rest, activity modification, anti- & ment including relative rest, NSAIDs, icing, \\
& undergo consecutive PRP injec- & inflammatory medication, physi- & rehabilitative exercise, and interval throw- \\
& tions, and postinjection MRI. & cal therapy, and interval throwing & ing program. Patients with full-thickness \\
& Patients with grade-1 or grade-3 & program. & UCL tears were excluded.
\end{tabular}

Intervention investigated

Outcome measures

Main findings

Level of

evidence

Validity score

(Downs and

Black) injuries, concomitant injuries to the extremity, or inability to comply with rehabilitation program were excluded.

Two 5- to 8-mL injections of leukocyte-rich PRP performed with ultrasound guidance $2 \mathrm{wk}$ apart. Rehabilitation program consisted of immediate bracing and a Kibler-integrated program focusing on kinetic chain strengthening. Interval throwing program began with the brace on and gradually worked toward throwing without a brace.

Return-to-participation and time to return to participation were followed. MRI performed to confirm diagnosis and $4 \mathrm{wk}$ after the beginning of treatment to evaluate healing. Outcomes were also considered separately for those with previous surgical intervention.

96\% of athletes without previous surgery were able to return to their previous level of competition. All athletes were cleared for participation at $6 \mathrm{wk}$, but mean time to return was longer due to specifics of sport seasons. MRI showed full reconstitution in $91 \%$ of these athletes. The only patient in this group who failed was able to return at $6 \mathrm{wk}$ but suffered another tear requiring surgery. Neither athlete with previous surgery was able to return to participation without surgical intervention.
One to three 3-mL injections of PRP along with 4-6 wk of progressive stretching and strengthening. An interval throwing program was initiated once athletes were asymptomatic and clinical exam was normal. Continued pain after 3 wk qualified patients for another injection.

Return-to-participation and time to return to participation were followed. Modified Conway Scale was used to classify the quality of return to sport. Outcomes are discussed separately based on performance level and location of tear.

Overall, 34\% of participants had an excellent outcome and $39 \%$ had a good outcome. Mean time to return to throwing was $5 \mathrm{wk}$ and mean time to return to competition was 12 wk (range 5-24 wk). $67 \%$ of professional players, $36 \%$ of college players, and $17 \%$ of high school players had excellent outcomes. $50 \%$ of position players had excellent outcomes and 38\% had good outcomes. All distal tears resulted in poor outcomes. $59 \%$ of proximal tears had good or excellent outcomes, whereas only $14 \%$ had poor outcomes. All grade- 1 tears had excellent $(47 \%)$ or good (53\%) outcomes.

One leukocyte-rich PRP injection of about $5 \mathrm{~mL}$ performed with ultrasound guidance combined with rehabilitation program. Rehabilitative exercise progressed as follows: range of motion, isometric strength, and concentric strength avoiding valgus forces. Stretching and valgus forces on the elbow allowed if pain free at week 5 .

Interval throwing program initiated at weeks $8-10$ and sport-specific training initiated at weeks 10-12.

Return to play and time to return to play were followed. KJOC and DASH scores collected every 4 wk during treatment and after return to sport. Ultrasound measurements collected every $4 \mathrm{wk}$ during treatment and at final follow-up.

After 70 wk of follow-up, $88 \%$ of athletes had returned to play at an average of $12 \mathrm{wk}$ (range 10-15 wk). Statistically significant improvements were seen in KJOC, DASH, and DASH-sports scores. Ultrasound measurements showed decreases in joint space opening and difference in joint space opening between involved and uninvolved limbs.

4

17 
Table 2 (continued)

\begin{tabular}{|c|c|c|c|}
\hline & Deal et al ${ }^{10}$ & Dines et $a^{6}$ & Podesta et $a^{8}$ \\
\hline Conclusion & $\begin{array}{l}\text { Two injections of leukocyte-rich } \\
\text { PRP can be used to treat primary } \\
\text { injuries to the UCL in high school } \\
\text { and college baseball and softball } \\
\text { players. Conservative treatment } \\
\text { with PRP may not be effective for } \\
\text { treatment of partial UCL tears in } \\
\text { athletes with previous surgical } \\
\text { intervention. }\end{array}$ & $\begin{array}{l}\text { PRP injections may be used in } \\
\text { combination with a rehabilitation } \\
\text { program to treat certain UCL } \\
\text { injuries in baseball players. } \\
\text { Grade- } 1 \text { injuries, proximal grade- } \\
2 \text { injuries, and high-level athletes } \\
\text { may benefit most from PRP } \\
\text { treatments. }\end{array}$ & $\begin{array}{l}\text { Leukocyte-rich PRP can be used to treat } \\
\text { partial tears of the UCL when combined } \\
\text { with an appropriate rehabilitative exercise } \\
\text { program. }\end{array}$ \\
\hline
\end{tabular}

Abbreviations: DASH, Disabilities of the Arm, Shoulder, and Hand; KJOC, Kerlan-Jobe Orthopaedic Clinic Shoulder and Elbow; MR, magnetic resonance; MRI, magnetic resonance imaging; NSAIDs, nonsteroidal anti-inflammatory drugs; PRP, platelet-rich plasma; UCL, ulnar collateral ligament.

rates are similar to those previously reported for all athletes seeking UCL reconstruction and are representative of the overall population sustaining UCL injuries. ${ }^{1}$ Clinicians must understand that these findings may not apply to athletes in other sports and should exercise clinical judgment based on the demands that will be placed on the UCL in such cases. For baseball and softball athletes, position players demonstrate better outcomes with conservative treatment than pitchers, likely due to exposure to fewer risk factors for UCL injury upon return to participation. 1,6,10

Along with clinical judgment based on the previously discussed patient- and injury-specific factors, clinicians should also consider the role of rehabilitation specialists in a conservative treatment plan. Extensive physical rehabilitation will be necessary and should be guided by an individual who is experienced in the management of athletic injuries. Rehabilitation specialists are especially important because rehabilitation should not be limited to the affected upper-extremity, but instead should focus on wholebody exercises and address movement patterns that may contribute to UCL injury. ${ }^{2,10}$ A typical postinjection protocol should consist of a period of relative rest and protection for the injured elbow while beginning to address strength and flexibility in other areas, followed by progressive stretching and strengthening of the upperextremity.2,6,8,10 Rehabilitation specialists should monitor and adjust exercises as necessary, determine the need for and duration of bracing, evaluate throwing mechanics and movement patterns, and select an appropriate progressive throwing program as an athlete prepares to return to competition.

Several limitations associated with this review should be addressed and considered when applying the results to clinical practice. None of the studies included in the review enrolled a true control group for comparison with the PRP group. Two studies required a failed trial of conservative treatment and interval throwing program as inclusion criteria, using each patient as his or her own control for comparison. This leaves the possibility for the natural time course to confound results, an issue that should be addressed in future studies by enrolling separate intervention and control groups. A second limitation is the lack of standardization and specific detail of rehabilitation plan utilized. None of the studies provide detailed explanations of their rehabilitation program, and results may be influenced by the postinjection process. Finally, all 3 studies employed different protocols for the formulation and administration of PRP injections. These studies differed on number of injections, volume of injections, inclusion or exclusion of leukocytes, and use of ultrasound guidance. These differences make direct comparison between studies difficult and negatively affect the reproducibility of the studies. Additionally, rationale for selection of a PRP protocol in these studies appears to be limited to clinical judgment and experience, manufacturer guidelines, or patient symptom reports. Future studies must include more specific characterizations and rationale for PRP formulations so work toward evaluating different formulations against one another can begin.

Future research should focus on evaluating rehabilitation and PRP protocols to allow for better comparison across studies. This should include detailed reports of rehabilitation protocols including timelines, specific rehabilitative exercises, bracing protocols, and throwing programs. PRP protocols should be evaluated to determine the effects of different formulations including volume administered, number and timing of injections, leukocyte-rich or leukocyte-poor formulations, and the use of ultrasound guidance for injections. There is also the possibility of publication bias in the literature, with positive outcomes more likely to be reported, skewing success rates. Therefore, higher level studies including case-control studies, prospective cohort studies, and randomized controlled trials must also be conducted to evaluate the efficacy of PRP. When possible, these studies should standardize patient selection, rehabilitation protocol, and PRP protocol.

In conclusion, the available research suggests that some athletes undergoing conservative treatment for partial UCL tears return to competition earlier and at higher rates when PRP is included in the treatment plan. ${ }^{6,8,10}$ Patient selection and injury severity greatly influence treatment outcomes, so clinicians must be mindful of these factors when educating athletes and making treatment recommendations. ${ }^{6,10}$ Athletes with proximal-based, low-grade injuries appear to be most likely to succeed in a conservative treatment plan with PRP.4,6-10 No adverse outcomes were reported with PRP injections, allowing clinicians to safely recommend use when conservative treatment is indicated. However, high-level evidence supporting the use of PRP for partial UCL tears is limited, and recommendations should be made on a case-by-case basis until further evidence is available.

\section{References}

1. Cain EL, McGonigle O. Return to play following ulnar collateral ligament reconstruction. Clin Sports Med. 2016;35(4):577-595. PubMed ID: 27543400 doi:10.1016/j.csm.2016.05.004

2. Patrick R, McGinty J, Lucado A, Collier B. Chronic UCL injury: a multimodal approach to correcting altered mechanics and improving healing in a college athlete-a case report. Int J Sports Phys Ther. 2016;11(4):614-626. PubMed ID: 27525185

3. Rettig AC, Sherrill C, Snead DS, Mendler JC, Mieling P. Nonoperative treatment of ulnar collateral ligament injuries in throwing athletes. Am J Sports Med. 2001;29(1):15-17. PubMed ID: 11206249 doi:10.1177/03635465010290010601

4. Kim NR, Moon SG, Ko SM, Moon WJ, Choi JW, Park JY. MR imaging of ulnar collateral ligament injury in baseball players: value 
for predicting rehabilitation outcome. Eur J Radiol. 2011;80(3):422426. PubMed ID: 21277722 doi:10.1016/j.ejrad.2010.12.041

5. Erickson BJ, Bach BR, Verma NN, Bush-Joseph CA, Romeo AA. Treatment of ulnar collateral ligament tears of the elbow: is repair a viable option? Orthop J Sports Med. 2017;5(1):2325967116682211. PubMed ID: 28203598 doi:10.1177/2325967116682211

6. Dines JS, Williams PN, El Attrache N, et al. Platelet-rich plasma can be used to successfully treat elbow ulnar collateral ligament insufficiency in high-level throwers. Am J Orthop. 2016;45(5):296-300. PubMed ID: 27552453

7. Frangiamore SJ, Lynch TS, Vaughn MD, et al. Magnetic resonance imaging predictors of failure in the nonoperative management of ulnar collateral ligament injuries in professional baseball pitchers. Am J Sports Med. 2017;45(8):1783-1789. PubMed ID: 28398820 doi:10. $1177 / 0363546517699832$
8. Podesta L, Crow SA, Volkmer D, Bert T, Yocum LA. Treatment of partial ulnar collateral ligament tears in the elbow with platelet-rich plasma. Am J Sports Med. 2013;41(7):1689-1694. PubMed ID: 23666850 doi:10.1177/0363546513487979

9. Ford GM, Genuario J, Kinkartz J, Githens T, Noonan T. Return-toplay outcomes in professional baseball players after medial ulnar collateral ligament injuries: comparison of operative versus nonoperative treatment based on magnetic resonance imaging findings. Am J Sports Med. 2016;44(3):723-728. PubMed ID: 26764237 doi:10. 1177/0363546515621756

10. Deal JB, Smith E, Heard W, O'Brien MJ, Savoie FH 3rd. Plateletrich plasma for primary treatment of partial ulnar collateral ligament tears: MRI correlation with results. Orthop J Sports Med. 2017; 5(11):2325967117738238. PubMed ID: 29164165 doi:10.1177/ 2325967117738238 